\title{
Endoscopic fringe projection for in-situ inspection of a sheet-bulk metal forming process
}

\author{
Steffen Matthias*a, Markus Kästner ${ }^{\mathrm{a}}$, Eduard Reithmeier ${ }^{\mathrm{a}}$ \\ ${ }^{\mathrm{a}}$ Institute of Measurement and Automatic Control, Leibniz Universität Hannover, Nienburger Str. 17, \\ 30167 Hannover, Germany
}

\begin{abstract}
Sheet-bulk metal forming is a new production process capable of performing deep-drawing and massive forming steps in a single operation. However, due to the high forming forces of the forming process, continuous process control is required in order to detect wear on the forming tool before production quality is impacted. To be able to measure the geometry of the forming tool in the limited space of forming presses, a new inspection system is being developed within the SFB/TR 73 collaborative research center. In addition to the limited space, the process restricts the amount of time available for inspection. Existing areal optical measurement systems suffer from shadowing when measuring the tool's inner elements, as they cannot be placed in the limited space next to the tool, while tactile measurement systems cannot meet the time restrictions for measuring the areal geometries. The new inspection system uses the fringe projection optical measurement principle to capture areal geometry data from relevant parts of the forming tool in short time. Highresolution image fibers are used to connect the system's compact sensor head to a base unit containing both camera and projector of the fringe projection system, which can be positioned outside of the moving parts of the press. To enable short measurement times, a high intensity laser source is used in the projector in combination with a digital micro-mirror device. Gradient index lenses are featured in the sensor head to allow for a very compact design that can be used in the narrow space above the forming tool inside the press. The sensor head is attached to an extended arm, which also guides the image fibers to the base unit. A rotation stage offers the possibility to capture measurements of different functional elements on the circular forming tool by changing the orientation of the sensor head next to the forming tool. During operation of the press, the arm can be travelled out of the moving parts of the forming press. To further reduce the measurement times of the fringe projection system, the inverse fringe projection principle has been adapted to the system to detect geometry deviations in a single camera image. Challenges arise from vibrations of both the forming machine and the positioning stages, which are transferred via the extended arm to the sensor head. Vibrations interfere with the analysis algorithms of both encoded and inverse fringe projection and thus impair measurement accuracy. To evaluate the impact of vibrations on the endoscopic system, results of measurements of simple geometries under the influence of vibrations are discussed. The effect of vibrations is imitated by displacing the measurement specimen during the measurement with a linear positioning stage. The concept of the new inspection system is presented within the scope of the TR 73 demonstrational sheet-bulk metal forming process. Finally, the capabilities of the endoscopic fringe projection system are shown by measurements of gearing structures on a forming tool compared to a CAD-reference.
\end{abstract}

Keywords: Fringe Projection, Vibrations, Endoscopy, Inspection, Metal Forming

\section{INTRODUCTION}

For many industrial applications, new manufacturing processes are tested in order to improve certain aspects of work pieces, such as weight or stability. One of these new processes is sheet-bulk metal forming, a combination of deep drawing and forming steps in a single process, which is being researched within the TR73 collaborative research center [1]. In order to ensure the quality of the work pieces, inspection of both work piece and tool is desired [2]. The quantitative data enables both the verification of work piece quality and adjustments of the process parameters. In the sheet-bulk metal forming process, high forming forces are applied to the tool making it necessary to continuously monitor the wear. However, due to the limited space available inside the forming press, commercially available measuring systems cannot fulfill the requirements. A new measurement system based on the fringe projection technique and endoscopy has been developed. Fringe projection profilometry is capable of obtaining areal measurements of the tool's geometry with measurement durations of less than one second. The system consists of a laser-based projector capable of projecting arbitrary patterns at high resolutions, which is housed in a base unit alongside an industrial camera. Flexible image fiber bundles guide both the projected pattern and the camera image to a compact sensor head. The compact design of the sensor head allows it to be positioned next to the forming tool. To be able to use the new

Optical Measurement Systems for Industrial Inspection IX, edited by Peter Lehmann,

Wolfgang Osten, Armando Albertazzi G. Jr., Proc. of SPIE Vol. 9525, 952513

(C) 2015 SPIE · CCC code: $0277-786 X / 15 / \$ 18 \cdot$ doi: 10.1117/12.2184746

Proc. of SPIE Vol. 9525 952513-1 
measurement system in the running process, the sensor head needs to be attached to a fast and precise positioning system to be able to remove the sensor head from the forming press in between measurements. The sum of the positioning and measuring time needs to be lower than the cycle time of the process. These timing restrictions impair the achievable accuracy of measurements. To reduce measurement times, the number of projected patterns needs to be reduced, resulting in a higher sensitivity to noise. One possibility to achieve lowest measurement and processing times is the usage of inverse fringe projection, which requires only a single image to detect geometry deviations. However, inverse fringe projection requires accurate positioning of the sensor head in the calibrated position, otherwise the sensitivity of measurements is reduced. Because of the length of the extended arm, the system is susceptible to vibrations. In addition, high positioning speeds are desired to meet the timing constraints, possibly leading to motion of the extended arm even if the axes are at halt. These vibrations affect both inverse and classical fringe patterns. The effect of the vibrations on the accuracy endoscopic system will be examined in the following sections.

\section{SHEET-BULK METAL FORMING}

Figure 1 (left) shows a drawing of the forming press, which is used for sheet-bulk forming experiments in subproject A7 within the collaborative research center TR73 [3]. Figure 1 (right) shows a drawing of the demonstrational forming tool for the bulk sheet metal forming process, which features complex gearing geometries. In order to achieve good results for the desired filigree geometries, high forming forces are required. To improve molding, the forming press setup shown in Figure 1 (left) is able to impose oscillations while applying forces. Especially the marked gearing elements are expected to be susceptible to wear and, therefore, should be inspected with the measurement system. Due to the mechanical design of the press, the available space in the forming press is very limited with a maximum height of less than $170 \mathrm{~mm}$ depending on the height of the forming tool. The construction of the moving parts of the press has a width of $1200 \mathrm{~mm}$ with the tool being at the center.

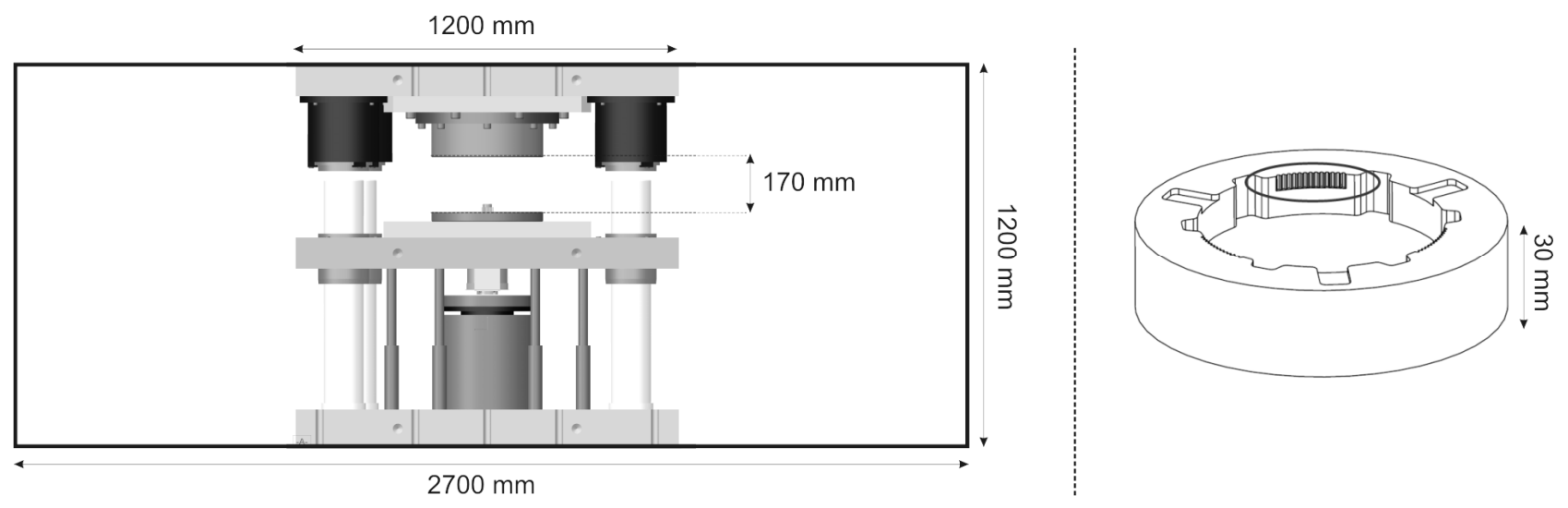

Figure 1. Schematic of the forming press (left), forming tool (right).

\section{INSPECTION SYSTEM}

As described in the previous section, the tool to be inspected is located at the center of the forming machine. To avoid damage to the measurement system, the sensor head has to be removed from the moving parts of the forming press. This results in a required length of $600 \mathrm{~mm}$ for the extended arm. It is required to be able to measure multiple gearing elements on the inner radius of the forming tool seen in Figure 1 (right). Therefore, a mechanism to rotate the sensor head will be required. The height above the forming tool is confined to a maximum of $150 \mathrm{~mm}$, which is the limit for the dimensions of both sensor head and the positioning equipment.

In order to meet the cycle time of the process, the time demand for the inspection of tool elements should in the range of a few seconds. This time needs to include the positioning of the sensor next to the forming tool, the measurement and the removal of the sensor from the press. Geometry deviations of more than $100 \mu \mathrm{m}$ need to be detected by the fringe projection system to avoid the production of damaged work pieces. 


\section{Measuring System}

The endoscopic fringe projection consists of a base unit, which is coupled to a compact sensor head using image fiber bundles to transport the projector and camera image. A schematic of the design can be seen in Figure 2. The base unit features a sealed enclosure to contain the projector's laser light source. A single mode DPSS laser supplied by Thorlabs with a wavelength of $532 \mathrm{~nm}$ and an optical output power of $40 \mathrm{~mW}$ is used as a light source in the pattern projector to complement with the maximum sensitivity wavelength of the camera sensor. To reduce laser speckle effects when emitting the light onto technical surfaces, a LSR-3005 laser speckle reducer by OptoTune is featured in the beam path. By modifying the optical path length over time the speckle is temporarily changing. By averaging the speckle effects over the camera's exposure time the speckle contrast can be greatly reduced. Following the speckle reducer, a beam homogenizer by SUSS Microoptics featuring two micro lens arrays, is inserted in order to achieve a homogenous illumination of fringe patterns. To generate the desired patterns, a digital micro-mirror device (DMD) with a resolution of 1024 by 768 pixels by Texas Instruments is used. The DLP Discovery 4100 kit is capable of projecting patterns with a gray-scale resolution of 8 bit per pixel at a rate of $271 \mathrm{~Hz}$. In combination with the high light intensities of the laser light source short a short measurement time of less than 3 seconds can be achieved.

In the camera part of the base unit a Point-Grey GRAS-50S5M-C gray-scale camera is used. To reduce the influence of ambient light on the measurements, an optical bandpass filter may be used which transmits only the wavelength of the employed laser. Using a hardware trigger connection enables precise synchronization of camera and projector.

For both camera and projector flexible image fibers by Fujikura are used. The diameter of the fibers is $1.7 \mathrm{~mm}$ at a resolution of 100.000 pixels. The minimum bending radius of the fibers is $200 \mathrm{~mm}$; lower radii can be realized by using a lower resolution fiber. To project the patterns onto the specimen and capture the image for the camera, gradient index lenses by Grintech are used. Compared to classical lenses, GRIN lenses have the advantage of having a plane image surface, which can be directly attached to the image fibers. Additionally, their dimensions are very compact with lengths less than $6 \mathrm{~mm}$ and a diameter of $2 \mathrm{~mm}$. Depending on the desired working distance, lenses with a focal length of $10 \mathrm{~mm}$ or $20 \mathrm{~mm}$ may be used. Both lenses are arranged in a triangulation angle using the current sensor head design, which exhibits a height of $100 \mathrm{~mm}$.

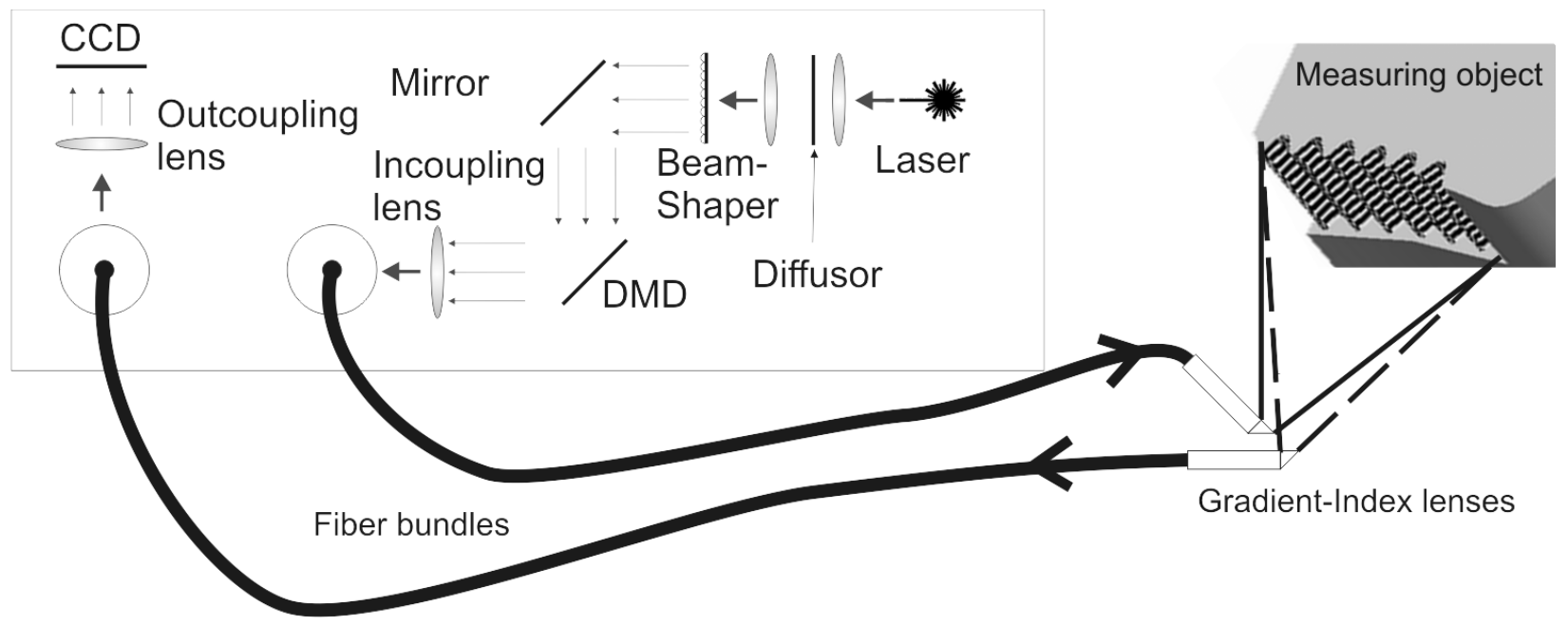

Figure 2. Schematic of the optical path of the endoscopic fringe projection system.

\section{Positioning System}

A graphical rendering of the devised positioning system is shown in Figure 3. The sensor head is attached to a rotation axis by Newport at the end of an extended arm. The axis has a height of $30 \mathrm{~mm}$ and will be used to select the elements to be measured on the inner radius of the forming tool while leaving enough space for the compact sensor head. For flexible positioning depending on the tool as well as to be able to lower the sensor head into the tool for an optimal working position, a vertical axis can adjust the vertical location of the sensor head. A horizontal axis moves the complete setup into the press to a position over the center of the forming tool. 
Due to the length of the extended arm, it is expected that the sensor head will be exposed to vibrations following the positioning step. Optionally, a damper might be applied to the far end of the extended arm, which can be used to mechanically connect to the inner body of the machine and thus reduce oscillations. In the following section, several evaluations will be carried out in order to assess the effect of the vibrations on the measurement quality.

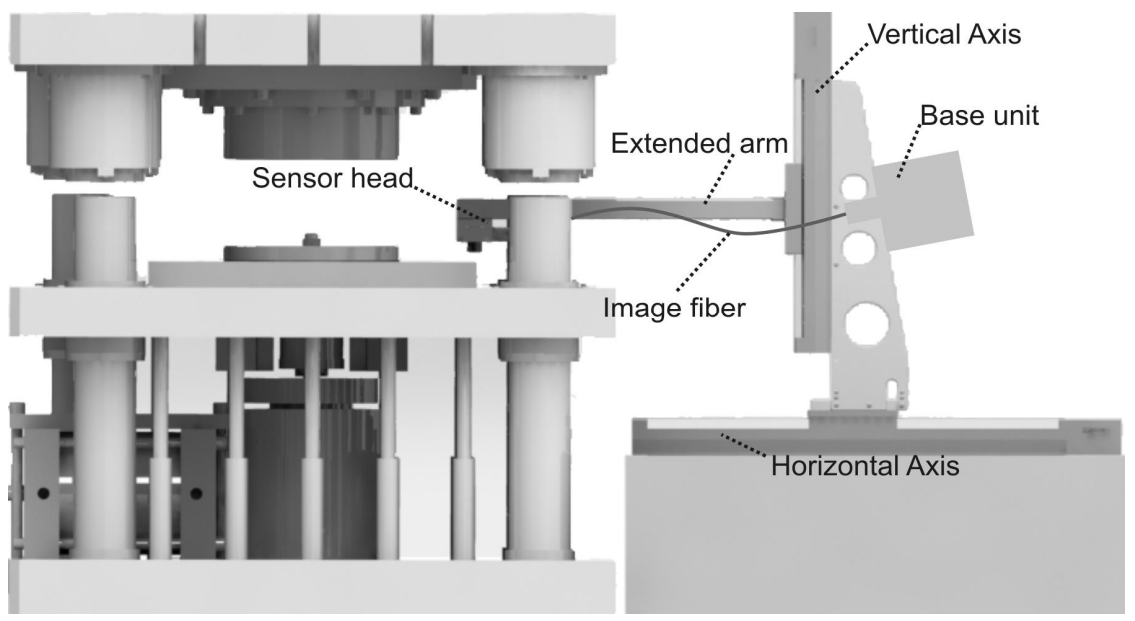

Figure 3. Design of the positioning system.

\section{FRINGE PATTERN EVALUATION}

To perform areal measurements with fringe projection profilometry, a sequence of fringe patterns is projected onto the specimen. Based on the deformation of these patterns from the camera perspective, the geometry of the specimen can be reconstructed in the form of a 3D point cloud. A phase-map is processed from the captured fringe patterns by an algorithm [4] with an appropriate system model, which is usually based on the pinhole camera model [5]. To obtain the $3 \mathrm{D}$ point cloud, a calibration is necessary, which describes the relation between phase and 3D coordinates using a suitable system model. Errors in measurements can arise from errors in the calibration as well as errors in the phase measurement step. To estimate effect of vibrations on the measurement quality, the calibration step is not a concern as it can be carried out in a static position. However, the vibrations will interfere with the phase measurement algorithms.

Generally, two different phase measurement approaches have been implemented to perform measurements. The first approach employs common absolute phase patterns, which are calculated from high-frequency phase-shift patterns in a phase-unwrapping step.

For the absolute phase measurement technique, phase-shift patterns are common with a shift count of four [4] for the highest frequency pattern. The evaluation of the relative phase value at each pixel $(\mathrm{u}, \mathrm{v})$ can be derived with Equation 1 from the four intensity images. A sequence of lower frequency patterns or binary patterns can be used to perform phase unwrapping to obtain an absolute phase-map. Because only the highest frequency shift carries the relevant phase information, the effect of vibrations on the binary or lower frequency patterns is not relevant for small displacements. However, if the amplitude of oscillations is too high, unwrapping errors will occur which lead to large errors in the reconstructed point clouds.

$$
\phi(\mathrm{u}, \mathrm{v})=\operatorname{atan} 2(I(u, v, 1)-I(u, v, 3), I(u, v, 2)-I(u, v, 4))
$$

Additionally, the inverse fringe pattern approach [6] has also been implemented for the system. By generating an adapted pattern for the desired specimen, no unwrapping of the pattern is required. Geometry deviations can be detected be comparing the captured camera image to the desired camera pattern, which has been defined in the initial simulation step. As only a single image is required and the comparison can be performed in pixel coordinates, measurement and processing time is greatly reduced compared to classical measurements. However, the generated pattern is only valid for the initially defined geometry and pose of sensor and specimen. Because only a single image is projected instead of a higher number of patterns, the phase noise is much higher than for the classical phase algorithms described earlier. This obviously reduces the sensitivity of the system. 


\section{IMPACT OF SENSOR HEAD DISPLACEMENT}

As previously described, vibrations of the sensor head are expected due to the long extended arm required. These vibrations interfere with both approaches in different ways. Generally, the motion of the sensor head leads to motion blur in the camera image. Choosing low exposure durations can reduce this effect. For the inverse fringe projection algorithm, the vibrations lead to a deviation from the previously defined sensor position. These deviations will lead to a global phase deviation. Thus, a threshold needs to be defined to account for the sensor displacements. Geometry deviations below this threshold cannot be detected. The classical fringe projection approach does not have the requirement of needing a predefined sensor position. However, the phase evaluation algorithms expect all phase shift images to be obtained at the same position.

Figure 4 shows a measurement of the gearing elements on the demonstrational forming tool to display the capabilities of the endoscopic system. The overall standard deviation compared to the CAD reference is $35 \mu \mathrm{m}$, with a maximum deviation of about $120 \mu \mathrm{m}$.

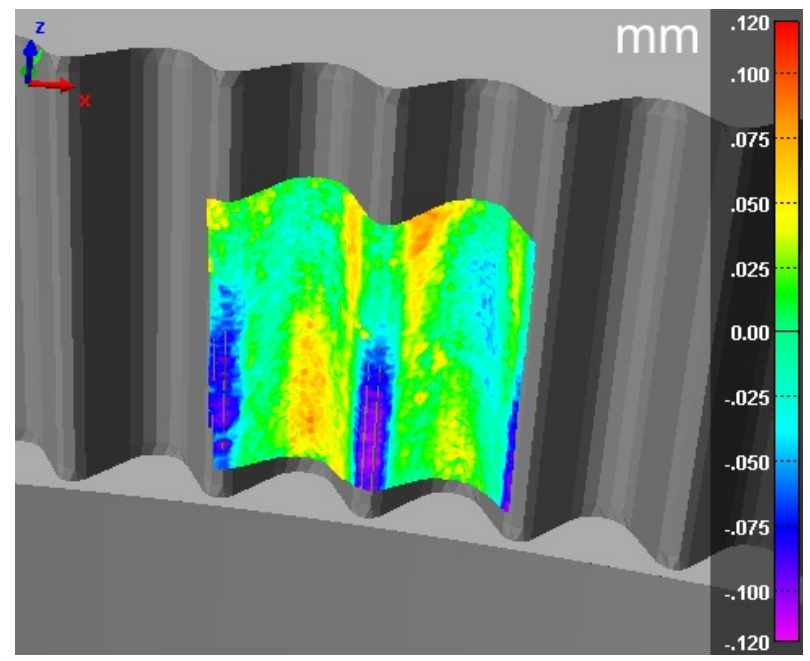

Figure 4. Deviation analysis of a measurement of the gearing elements on the forming tool.

While the geometry of the specimen will have a great influence on the effect of sensor displacement while measuring, the impact will first be studied by measurements of a specimen with a planar surface, followed by a simulation of measurements with more complex geometries.

\section{Measurements}

Figure 5 shows results of measurements with simulated vibrations of different amplitude. In order to emulate the vibrations, the specimen has been moved with a linear axis in the direction of the surface normal between capturing the individual images of the phase-shift patterns. To demonstrate the reduced sensitivity due to vibrations, a planar surface with a spherical cavity with a depth of approximately $90 \mu \mathrm{m}$ and a diameter of $0.6 \mathrm{~mm}$ has been selected as a specimen. The surface of the specimen is covered with diffuse spray to improve its optical characteristics. It needs to be noted that the results show the maximum error case as each phase image has been captured at the position of maximum displacement for the given oscillation amplitude. For each measurement, the number of 3D surface points is approximately 18.000 .

For an oscillation amplitude of $0 \mu \mathrm{m}$, e.g. a measurement without displacement, the spherical cavity is clearly visible in the lower part of the image. The measurement shows deviations in the range of $-20 \mu \mathrm{m}$ to $20 \mu \mathrm{m}$ for the planar area. As the amplitude increases, periodic structures can be seen in the measurements, which arise from errors in the absolute phase-map. For an amplitude of $60 \mu \mathrm{m}$, the cavity is still visible. However, the deviations from phase-map errors are already of similar intensity.

While the relative error in the relative phase measurement step may be reduced by using higher frequency patterns, unwrapping errors will occur. 

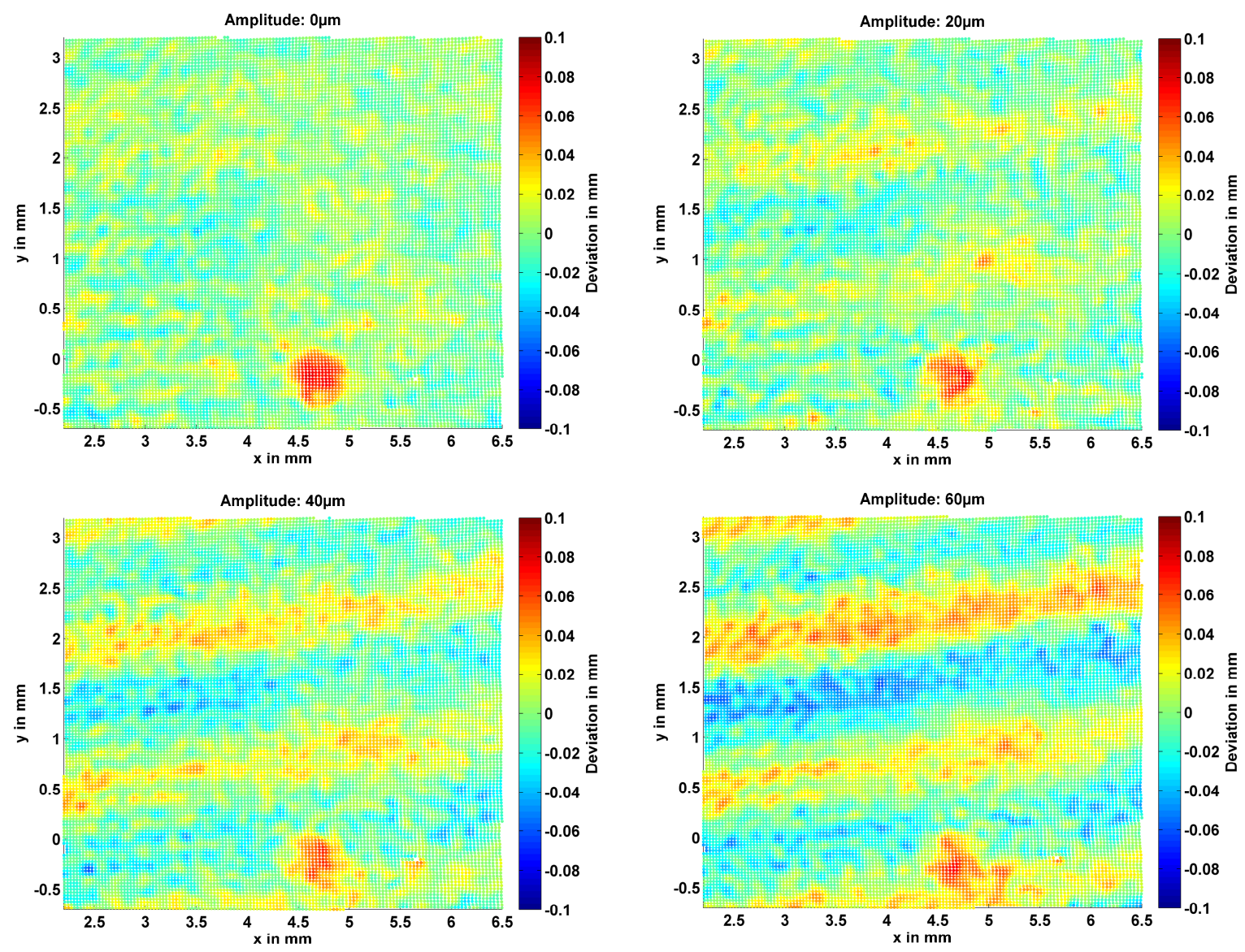

Figure 5. Measurements of a specimen at different emulated oscillation amplitudes.

\section{Simulation}

A virtual fringe projection system based on the system models employed also for the evaluation of phase-map data will be used for the simulation of more complex geometry. The virtual system uses calibration data gathered from the real fringe projection system and is thus able to model the optical parameters of the system. It is capable of both performing virtual measurements of a given CAD geometry as well as simulating inverse projector patterns as described in Section 4. However, some negative effects, such as imaging noise and motion blur, are currently not modeled.

Figure 4 shows a rendering of the CAD geometry used for the simulation of inverse patterns and measurement. As motion is expected mostly in z-direction, e.g. vertically inside the press, the motion vector of vibrations has been defined to $50 \mu \mathrm{m}$ motion in direction of the z-axis (parallel to gearings) and $10 \mu \mathrm{m}$ motion in direction of the $\mathrm{x}$-axis (sideward). An example of the inverse pattern can be seen in Figure 1 (left), while the simulated deviations in projector pixels are given on Figure 6 (right). 

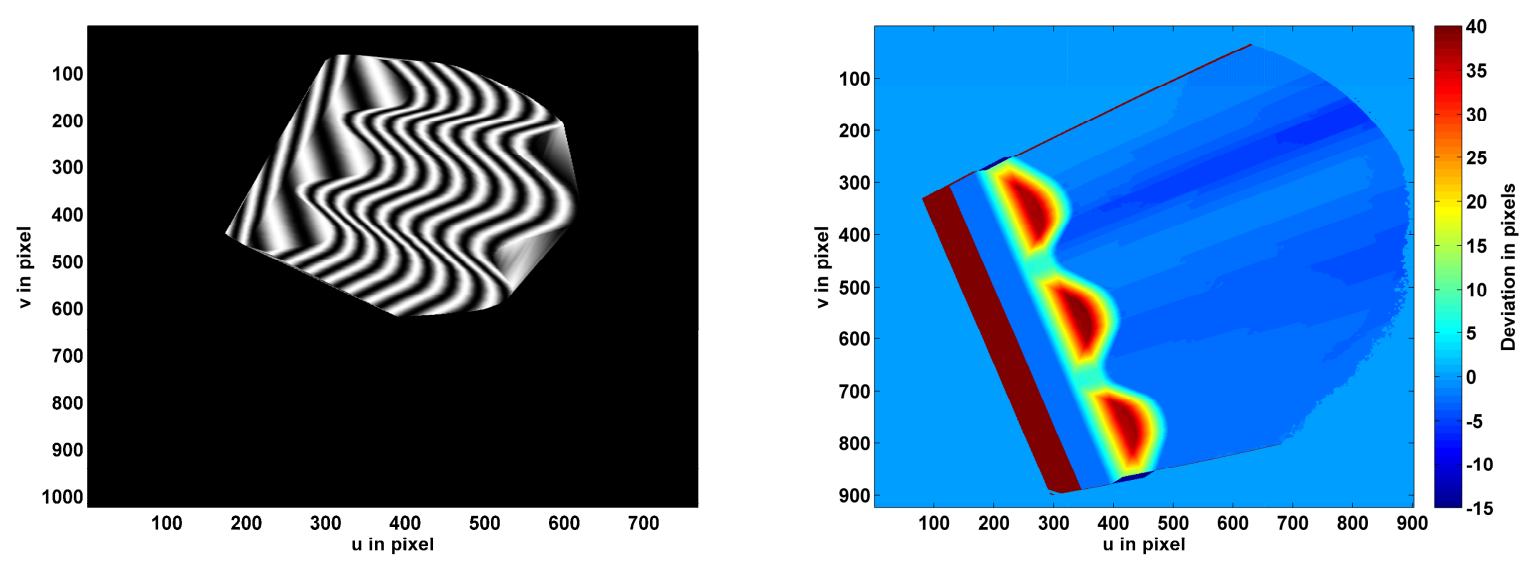

Figure 6. Inverse projector pattern for gearing geometry (left), simulated deviations from sensor displacement (right).

Metric information is not given as for the inverse approach usually only a non-metric threshold is defined above which deviations are regarded as critical. It can be seen that the largest deviations are seen at the lower end of the gearings. Deviations on the gearings itself are much lower. This is due to the direction of movement being mostly in z-direction to which the gearings are symmetric. Only the smaller movement in the direction of the $\mathrm{x}$-axis introduces deviations on the gearings. Therefore, the actual sensitivity when inspecting the demonstrational forming tool is expected to be much higher than estimated from the previous measurements. However, it needs to be noted that the actual deviations are expected to be higher than in the simulation due to inaccuracies in the calibration data and phase noise.

Pösch established the approach of a sensitivity map [7], which can give an estimation of the scale of geometry deviations detected with inverse fringe projection. Using the results from the above simulations and actual information of sensor displacement, it could be extended with an uncertainty estimation.

\section{CONCLUSION AND FUTURE WORK}

A novel design for a tool inspection for the application in running metal forming processes has been presented. The proposed design of the sensor head and positioning system has a height of only $130 \mathrm{~mm}$. Several measurements and simulations have been conducted in order to assess the effect of vibrations of the sensor head on measurement accuracy. It could be shown that the effect is dependent on the geometry of the specimen and the direction of the sensor head movement. A worst-case estimation based on the measurements showed that geometry deviations on planar surfaces of $100 \mu \mathrm{m}$ could still be detected with oscillation amplitudes of up to $60 \mu \mathrm{m}$ for phase-shift patterns.

In order to estimate the influence of vibrations, which may arise from the length of the positioning system's arm, simulations as well as measurements have been demonstrated. For vibrations with amplitudes of less than 50 um, the system is still able to detect deviations of $100 \mu \mathrm{m}$ on planar surfaces.

Different acceleration profiles will be evaluated to have as less vibrations as possible while maintaining a fast positioning process. In order to obtain exact information on the sensor head movement, laser tracker measurements will be carried out. Based on this information and the desired model geometry, the simulation of inverse fringe patterns will be extended to give an estimated sensitivity for each measurement point. Using Fourier analysis, vibrations could also be estimated during measurements [8]. Finally, with a more advanced sensor head design, the height of the inspection system will be reduced in the future.

\section{ACKNOWLEDGEMENT}

The authors would like to thank the German Research Foundation (DFG) for funding the project B6 "Endoscopic geometry inspection" within the Collaborative Research Center (CRC) / TR 73. 


\section{REFERENCES}

[1] Merklein, M, Allwood, J. M., Behrens, B. , Brosius, A. , Hagenah, H. , Kuzman, K. , Mori, K. , Tekkaya, E. and Weckenmann, A., "Bulk forming of sheet metal," CIRP Annals-Manufacturing Technology, vol.61(2), pp. 725-745.

[2] Ohrt, C., Hartmann, W., Weickmann, J., Kästner, M., Weckenmann, A., Hausotte, T. and Reithmeier, E., "Holistic Measurement in the Sheet-Bulk Metal Forming Process with Fringe Projection," Key Engineering Materials 504, pp. $1005-1010$

[3] Behrens, B., Hübner, S., Vucetic, M., Koch, S., Denkena, B., Grove, T., Lucas, H., Tillmann, W., Stangier, D., Hausotte, T., Loderer, A., "Prozessauslegung und Untersuchung von geschliffenen und CrAlN / CrAlCNbeschichteten Werkzeugoberflächen mittels schwingungsüberlagertem Flachstauchversuch für die Blechmassivumformung," Tagungsband zum 18. Workshop Simulation in der Umformtechnik \& 3. Industriekolloquium Blechmassivumformung 2015 - DFG Transregio 73, Aachen: Shaker Verlag, pp. 173 - 192, (2015).

[4] Peng, T., "Algorithms and models for 3-D shape measurement using digital fringe projections," Thesis, University of Maryland, (2007).

[5] Hartley, R. and Zisserman, A., "Multiple View Geometry in Computer Vision," Cambridge University Press, ISBN: 0521540518.

[6] Kalms, M. K., Osten, W. and Jueptner, W., "Inverse projected-fringe technique with automatic pattern adaption using a programmable spatial light modulator," International Conference on Applied Optical Metrology, International Society for Optics and Photonics, (1998).

[7] Pösch, A., Vynnyk, T., and Reithmeier, E., "Using inverse fringe projection to speed up the detection of local and global geometry defects on free-form surfaces," SPIE Optical Engineering+ Applications, International Society for Optics and Photonics, (2012).

[8] Spagnolo, G. S., Paoletti, D. and Ambrosini, D., "Vibration monitoring by fiber optic fringe projection and Fourier transform analysis," Optics communications 139.1, pp. 17-23, (1997). 